\title{
HIV and HIV Genome
}

National Cancer Institute

\section{Source}

National Cancer Institute. HIV and HIV Genome. NCI Thesaurus. Code C18837.

1A1; Basic Sciences Research (includes vaccine and chemotherapy development);

Biomedical Research; HIV and HIV Genome (structure, function) 